\title{
Hydraulic Balance Debugging Method for Air Conditioning Water System
}

\author{
Qin $\mathrm{Hu}$ and Zhongyuan Yuan* \\ School of Mechanical Engineering, Southwest Jiaotong University, Chengdu, Sichuan, 610000, China
}

\begin{abstract}
By installing the balance valve in each user branch, the pressure at both ends of the balance valve is obtained in real time by using the pressure measuring instrument, and then the hydraulic balance calculation program is written by combining the fluid network matrix and MATLAB software to calculate the balance valve opening value under the design flow of each user branch. This calculation program only needs to input a few simple data to calculate the opening of each balance valve when each branch reaches hydraulic balance. Experimental results show that the method can achieve good results in hydraulic balance debugging.
\end{abstract}

\section{Introduction}

In recent years, with the rapid development of China's economy, people's living standards continue to improve, the people are more and more attention to the quality of life, the comfort of the living environment is also higher and higher requirements. The most commonly used to create the comfort of indoor environment is the air conditioning system. Most air conditioning systems can be roughly divided into wind system and water system, in which the air conditioning water system bears the important responsibility of transporting refrigerant and transferring cooling capacity. In order to ensure that the air conditioning system can operate normally under design conditions, we must deal with the most common problem of hydraulic imbalance in the air conditioning water system. The inconsistency between the actual flow and the designed flow of each branch in the fluid pipe network system is called the hydraulic imbalance of the branch. The hydraulic imbalance of the pipelines in the water system of air conditioning will lead to the unbalance of cold and heat in the air conditioning area, which has a great impact on the comfort of users. Hydraulic imbalance will make the flow rate of each pipeline inconsistent with the design flow rate, so that the flow rate of some pipelines is too large, resulting in the phenomenon of wasted energy. In the past, many common hydraulic balance debugging methods, such as proportion method and compensation method, were difficult, time-consuming, too professional and not intuitive. The debugging method proposed in this paper is to directly calculate the opening value of the balance valve of each branch by using the calculation program after the input of some simple data. It is only necessary to manually adjust the balance valve of each branch to the calculated value. The debugging method is simple, quick and easy to operate. This paper establishes and verifies the calculation program of hydraulic balance debugging by combining numerical simulation with experimental research. The experimental results show that the debugging method is effective.

\section{$2 \quad$ Hydraulic balance debugging method}

The hydraulic balance debugging method proposed in this paper is mainly divided into three steps.

\subsection{Number the branches}

The schematic diagram of the air conditioning water system is as follows.

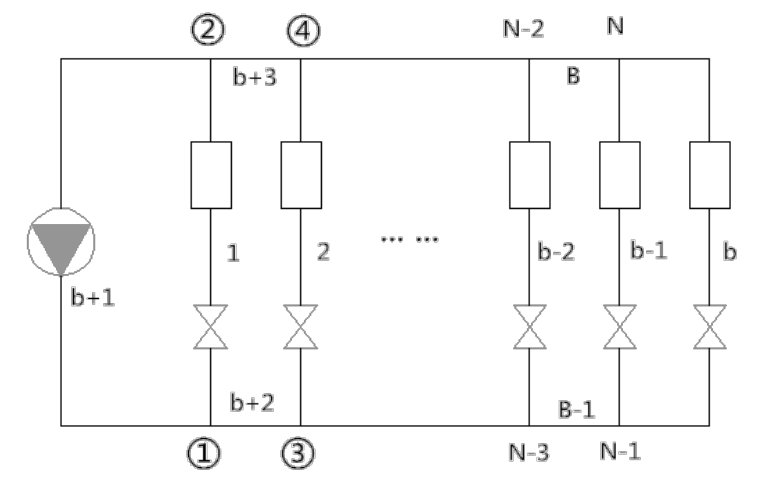

Figure 1. Schematic diagram of hydraulic pipe network for air conditioning water system.

Set the number of nodes as $\mathrm{N}$, the number of branches as B and the number of user branches as $b$, then the number of branches except user branches is B-b. First of all, each node of the whole air-conditioning water system is numbered from (1) to N. Then numbered each branch. The numbering principle is to first number the

${ }^{*}$ Corresponding author's e-mail: Zhongyuan.yuan@home.swjtu.edu.cn 
user branch equipped with the balancing valve, and then number the other trunk branches in the system. The user branch is numbered from 1 to $b$, and the rest are $b+1$ to $B$. According to this principle, the number satisfies the rule of $3 \mathrm{~N}=2 \mathrm{~B}$.

\subsection{Calculating the impedance coefficient $S$ of each branch when the balance valve is fully open}

When the whole air conditioning water system pipe network is established, the impedance of other parts is determined except the adjustable valve door. At this time, only the adjustable valve opening can affect the impedance of each branch. When the valve opening changes, the impedance of each branch will also change.

\subsubsection{Adjusting the branch balance valve to full open state and building the system of equations.}

1) Calculating the branch flow of each user branch. The flow column vector $Q$ of each branch can be divided into $Q_{l}$ and $Q_{t}$. $Q_{l}$ represents the flow of each user branch, and $Q_{t}$ represents the flow of all branches except the user branch. The differential pressure gauge is installed at the balance valve of each user branch to measure the pressure difference at both ends of the balance valve in real time. According to formula (1), the flow $Q_{l}$ of each user branch can be calculated. $Q_{l}$ is a column vector of b rows and 1 column.

$$
Q=K \sqrt{\Delta P}
$$

In formula (1), $Q$ is flow rate $(1 / \mathrm{h}) ; K$ is the flow coefficient; $\triangle P$ is the differential pressure $(\mathrm{kPa})$ at both ends of the balancing valve.

According to the flow coefficient value corresponding to some specific opening degrees given by the balance valve manufacturer, the relation between the balance valve opening degree $x$ and the flow coefficient $K$ can be obtained by polynomial fitting as follows:

$$
\begin{gathered}
K=-1.472 \mathrm{x}^{3}+19.04 x^{2}+9.659 x+4.716 \\
K=-8.8 \mathrm{x}^{3}+80.024 x^{2}-61.169 x+65.729
\end{gathered}
$$

Formula (2) is the relation between the flow coefficient $K$ of the balance valve of DN15 pipe diameter and the opening degree $x$ of the balance valve; Formula (3) is the relation between balance valve flow coefficient $K$ of DN20 pipe diameter and balance valve opening degree $x$.

In formula (2) and (3), $K$ is the flow coefficient. When the balance valve is fully closed, $K$ is 0 , and the rest is calculated by the above relationship. $x$ is the opening degree of the balance valve. When the balance valve is fully open, $x$ is 4 and when it is fully closed, $x$ is 0 .

2) Listing the incidence matrix and basic loop matrix of the fluid network. The construction principle of the incidence matrix $A$ is as follows: take the node as the row and all the branches as the column. When the water flow in the branches points to the node, take -1 ; when it leaves the node, take 1 ; when it is not connected to the node, take 0 .

The basic loop matrix $B_{f}$ is constructed according to the following principles: firstly, the fluid network structure is divided, the user branch is the chain branch, and the rest is the tree branch. Multiple tree branches and a chain branch constitute a basic loop. Before construction, it is necessary to identify the total number of the basic loop, take the basic loop as the row, and all the branches as the column. When the flow direction of the branch is the same as the direction of the basic circuit, take 1 ; when it is opposite, take -1 ; when the branch is not in the basic circuit, take 0 .

After the construction is complete, the correlation matrix $A$ is $\mathrm{N}-1$ rows and $\mathrm{B}$ columns, and the basic loop matrix $B_{f}$ is b rows and $\mathrm{B}$ columns.

3) Solving flow rate of tree branch. The chain branch flow $Q_{l}$ can be calculated from formula (1), and according to formula (4), the tree branch flow $Q_{t}$ can be calculated. $Q_{t}$ is a column vector of B-b rows and 1 column.

$$
A \bullet Q=0
$$

4) Building the system of equations. According to the fluid network analysis theory, there are:

$$
B_{\mathrm{f}} \bullet\left|Q_{m}\right| \bullet Q_{m} \bullet S=B_{\mathrm{f}} \bullet D
$$

In formula (5), $Q_{m}$ is a diagonal matrix of B rows and $\mathrm{B}$ columns, and the values on the main diagonal are the flow of each branch $(1 / \mathrm{h}) ; D$ is the power head of the pump $(\mathrm{kPa})$, and $D$ is a column vector of B rows; $S$ is a column vector of $\mathrm{B}$ rows composed of the branch impedance coefficients.

It can be seen from figure 1 that two opposite branches, such as branch $b+1$ and branch $b+2$, have the same pipe diameter and length, so it can be considered that the impedance of branch $b+1$ and branch $b+2$ is approximately equal. In the same way, the impedance of two opposite branches can be considered equal. Then, the number of the same impedance branches in the impedance column vector $S$ is (b-2), so the unknown number of the impedance column vector $S$ is B-(b-2).

In formula (5), the number of equations is $b$, and the number of unknowns is less than the number of equations, so the equations cannot be solved, and other equations need to be constructed to solve the equations.

2.2.2. Closing the balance valve of a branch and building the system of equations. After arbitrarily closing a branch balancing valve, repeat the steps in 2.2.1. After a user branch is closed, the remaining branches have no valve action, so the impedance of the remaining branches can be regarded as unchanged except the closed branch.

1) Calculating the branch flow of each user branch. After closing a branch balance valve, the number of nodes in the water system pipe network is $\mathrm{N}-2$, the number of branches is B-1, the number of user branches is $b-1$, and the number of other branches except the user 
branch is B-b. At this time, the flow column vector $Q_{g}$ of each branch can be divided into $Q_{l g}$ and $Q_{t g}$. $Q_{l g}$ represents the flow of each user branch after closing a branch balance valve, and $Q_{t g}$ represents the flow of other branches except the user branch.

The pressure difference at both ends of the balancing valve is measured according to the pressure difference sensor. The flow rate $Q_{l g}$ of each user branch can be calculated according to formula (1). $Q_{l g}$ is a column vector of $b-1$ rows and 1 column.

2) Listing the incidence matrix and basic loop matrix of the fluid network. In the same way, building a new incidence matrix $A_{\mathrm{g}}$ with N-3 rows and B-1 columns and a new basic loop matrix $B_{f g}$ with b-1 rows and B-1 columns.

3) Solving flow rate of tree branch. Calculating the flow $Q_{t g}$ of the tree branch according to formula (4). $Q_{t g}$ is a column vector of B-b rows and 1 column. Combining the column vector $Q_{l g}$ and the column vector $Q_{t g}$ can immediately obtain the flow $Q_{g}$ of each branch after closing a certain branch balancing valve. $Q_{g}$ is a column vector of B rows and 1 column.

4) Building the system of equations. According to the fluid network analysis theory, there are:

$$
B_{\text {fg }} \bullet\left|Q_{m g}\right| \bullet Q_{m g} \bullet S_{g}=B_{f g} \bullet D_{g}
$$

In the formula (6), the impedance of the closed branch in $S_{g}$ is 0 , and the impedance of the other branches is the same as the impedance $S$ of each branch when the balance valve is fully opened. Therefore, the number of unknowns in formula (6) is B-(b-2)-1, and the number of equations is $b-1$.

\subsubsection{Calculating the impedance by combining equations. By combining the two equations in the above calculation, the impedance $S$ of each branch can be obtained immediately.}

1) Calculating the impedance of each branch when the balance valve is fully open. Simultaneous equations (5) and (6), the number of equations at this time is $2 b-1$, and the number of unknowns is $\mathrm{B}-(\mathrm{b}-2)$. Since $3 \mathrm{~N}=2 \mathrm{~B}$ and $b=B-(N-1), 2 b-1=B-(b-2)$, that is, the number of equations is equal to the number of unknowns, and the equations are solvable. Simultaneously, the two equations can be solved to obtain the impedance value $S$ of each user branch when the balance valve is fully opened.

2) Calculating the impedance of all components except the balance valve in the user branch. According to the theory of fluid mechanics, the following formula can be used for calculation.

$$
S_{\mathrm{qy}}=S-S_{f, q k}
$$

In formula (7), $S_{q y}$ is the impedance of the other parts in the user branch except the balance valve; $S$ is the total impedance of each user branch when the balance valve is fully open; $S_{f, q k}$ is the impedance of the balance valve when the balance valve is fully open, which can be calculated according to formula (8).

$$
S_{f, q k}=\left(\frac{1}{K_{\mathrm{qk}}}\right)^{2}
$$

In formula (8), $K_{q k}$ is the flow coefficient when the balance valve is fully open, which can be calculated according to equations (2) and (3).

\subsection{Calculating the opening value of each branch balance valve under design condition}

\subsubsection{Building the impedance calculation equations} of the user branch. After the equations are constructed, the impedance of each user branch can be calculated.

1) Calculating the impedance of each branch when the balance valve is fully open. The flow rate of each branch in the design condition can be determined by the user data.

2) Listing the incidence matrix and basic loop matrix of the fluid network. The incidence matrix $A_{s j}$ and basic loop matrix $B_{f s j}$ in the design condition are constructed in the same way. Since the total number of pipe network structure and branches in the design state is consistent with the total number of pipe network structure and branches when the balance valve is fully open, the incidence matrix $A_{s j}$ and the basic loop matrix $B_{s j}$ in the design state are consistent with the association matrix $A$ and the basic loop matrix $B_{f}$.

3) Solving flow rate of tree branch. After the design flow of each user branch is known, the tree branch flow rate can be calculated according to formula (4).

4) Building the system of equations.

$$
B_{\mathrm{f} s j} \bullet\left|Q_{\mathrm{sj}}\right| \bullet Q_{s j} \bullet S_{s j}=B_{f s j} \bullet D
$$

The impedance of all branches in the fully open state of the balance valve of the user branch has been calculated in step 2.2.3. The impedance of the tree branch can be considered unchanged because there is no valve action in the tree branch. So the unknown part of $S_{s j}$ is only the impedance of the user branch, namely the chain branch. In equation (9), the number of equations is $b$, the number of unknowns is $b$, the number of unknowns is equal to the number of equations, so the equations can be solved. After solving the equations, the impedance of the user branch in the design condition can be obtained.

\subsubsection{Calculating the opening value of balance valve of the user branch in the design condition.}

The impedance composition of the user branch is as follows:

$$
S_{l, s j}=S_{f, s j}+S_{q y}
$$

In formula (10), $S_{l, s j}$ is the impedance of the user branch in the design condition; $S_{f, s j}$ is the impedance of the balance valve of the user branch in the design condition; $S_{q y}$ is the impedance of the other parts of the user branch except the balance valve. 
The impedance of the balance valve of the user branch in the design condition can be calculated from equation (10). The flow coefficient of the balance valve is calculated according to the following formula.

$$
K_{s j}=\frac{1}{\sqrt{S}_{f, s j}}
$$

According to formula (11), the flow coefficient of the balance valve of each user branch under the design conditions can be calculated. Then the balance valve opening $x$ of user branches with different pipe diameters in the design conditions can be calculated according to formula (2) and (3).

After obtaining balance valve opening value $x$ of each user branch in the design condition, the hydraulic balance debugging of the whole water system can be completed by manually adjusting the balance valve of each branch to its corresponding calculated value.

\section{Experimental verification}

Using MATLAB software to write the above calculation process into a calculation program that only needs to input a few simple raw data to directly calculate the opening degree of each user's branch balance valve under design conditions. An experimental bench was set up to simulate the operation of a normal air conditioning water system, and the written calculation program was applied to the experimental bench to verify the feasibility of the hydraulic balance debugging method.

\subsection{Basic information of experimental bench}

The equipment of this experimental bench mainly includes water pump, flow meter, pressure gauge, balance valve, lock valve, water pipe, pressure sensor and frequency converter. The experimental bench is designed with a total of 6 branches, which respectively simulate 6 air conditioning users. The lock valve which can fix the opening degree is used to simulate the resistance of air conditioning equipment that replaces the users of the actual air conditioning system. A static balance valve is installed on the return pipe to adjust the resistance and flow of each pipe. Balance valve is an important part of hydraulic balance debugging of air conditioning water system.

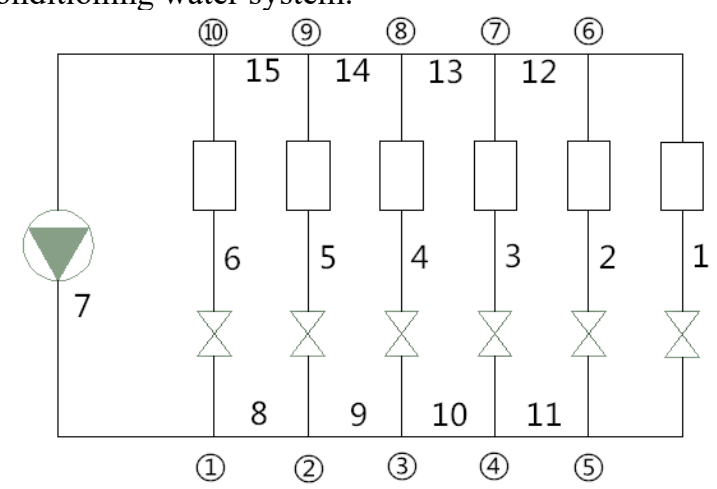

Figure 2. Schematic diagram of experimental bench system.

In this experiment bench, the number of nodes $\mathrm{N}=10$, the number of branches $\mathrm{B}=15$, and the number of user branches $b=6$. Branch 1-3 is DN15 pipe diameter, and branch 4-6 is DN20 pipe diameter.

\subsection{Putting experimental data into the calculation program}

Numbering the branches and nodes of the experimental bench. The numbering results are shown in figure 2 . Adjusting the balance valve of the 6 user branches of the experimental platform to the fully open state, then record the opening value of the balance valve $x$, the pressure difference $\Delta P$ across the balance valve, and the pump head $D$. Closing user branch 1 and recording the opening value $x_{g}$ of each balancing valve and the differential pressure $\Delta P_{g}$ across the balancing valve at this time. Fill in the following table with the numbers of the nodes and branches and the recorded data. In the characteristic column of user branch pipe diameter in Table 1, 1 means DN15 pipe diameter, 2 means DN20 pipe diameter.

Table 1. The input values required by the calculation program.

\begin{tabular}{|c|c|c|c|c|c|c|c|c|c|}
\hline $\begin{array}{c}\text { Start } \\
\text { number }\end{array}$ & $\begin{array}{c}\text { End } \\
\text { number }\end{array}$ & $\begin{array}{l}\text { Branch } \\
\text { number }\end{array}$ & $\begin{array}{c}\text { Characte } \\
\text { ristic } \\
\text { column } \\
\text { of user } \\
\text { branch }\end{array}$ & $\begin{array}{c}\text { Differential } \\
\text { pressure } \\
\triangle P(\mathrm{kPa})\end{array}$ & $\begin{array}{c}\text { Opening } \\
\text { value of } \\
\text { balance } \\
\text { valve } \\
x\end{array}$ & $\begin{array}{c}\text { Pump } \\
\text { head } \\
D(\mathrm{kPa})\end{array}$ & $\begin{array}{c}\text { Flow } \\
\text { rate in } \\
\text { design } Q \\
(1 / h)\end{array}$ & $\begin{array}{l}\text { Differential } \\
\text { pressure } \\
\triangle P_{g}(\mathrm{kPa})\end{array}$ & $\begin{array}{c}\text { Opening } \\
\text { value of } \\
\text { balance } \\
\text { valve } \\
x_{g}\end{array}$ \\
\hline 5 & 6 & 1 & 1 & 2.9 & 4.0 & 0.0 & 400.0 & 0.0 & 0.0 \\
\hline 5 & 6 & 2 & 1 & 3.1 & 4.0 & 0.0 & 400.0 & 4.1 & 4.0 \\
\hline 4 & 7 & 3 & 1 & 3.3 & 4.0 & 0.0 & 400.0 & 4.2 & 4.0 \\
\hline 3 & 8 & 4 & 2 & 2.7 & 4.0 & 0.0 & 900.0 & 3.0 & 4.0 \\
\hline 2 & 9 & 5 & 2 & 3.4 & 4.0 & 0.0 & 900.0 & 3.9 & 4.0 \\
\hline 1 & 10 & 6 & 2 & 3.7 & 4.0 & 0.0 & 900.0 & 4.2 & 4.0 \\
\hline 10 & 1 & 7 & 0 & 0.0 & 0.0 & 66.5 & 0.0 & 0.0 & 0.0 \\
\hline
\end{tabular}




\begin{tabular}{lccccccccc}
1 & 2 & 8 & 0 & 0.0 & 0.0 & 0.0 & 0.0 & 0.0 & 0.0 \\
2 & 3 & 9 & 0 & 0.0 & 0.0 & 0.0 & 0.0 & 0.0 & 0.0 \\
3 & 4 & 10 & 0 & 0.0 & 0.0 & 0.0 & 0.0 & 0.0 & 0.0 \\
4 & 5 & 11 & 0 & 0.0 & 0.0 & 0.0 & 0.0 & 0.0 & 0.0 \\
6 & 7 & 12 & 0 & 0.0 & 0.0 & 0.0 & 0.0 & 0.0 & 0.0 \\
7 & 8 & 13 & 0 & 0.0 & 0.0 & 0.0 & 0.0 & 0.0 & 0.0 \\
8 & 9 & 14 & 0 & 0.0 & 0.0 & 0.0 & 0.0 & 0.0 & 0.0 \\
9 & 10 & 15 & 0 & 0.0 & 0.0 & 0.0 & 0.0 & 0.0 & 0.0 \\
\hline
\end{tabular}

After the calculation program has been written, we only need to manually input simple data such as the branch number of each node, the differential pressure of the user branch, and the opening value of the balance valve. After being read by the calculation program, the program can directly calculate the opening value of the balance valve of the branch users in the design conditions. After reading the data in Table 1, the output of the calculation program is $x_{1}=2.30, x_{2}=2.26, x_{3}=$ $2.22, x_{4}=2.65, x_{5}=2.30, x_{6}=2.24$, which represent the opening value of balance valve of user branch 1-6 in the design conditions.

Manually adjust the balance valve of each user branch to the calculated value to complete the hydraulic balance debugging of the water system. After the commissioning is completed, measure and record the differential pressure across the balance valve of each user branch. According to known opening value of balance valve and differential pressure, the actual flow rate of each branch can be calculated according to formula (1).

Table 2. Results of hydraulic balance debugging.

\begin{tabular}{ccccccc}
\hline $\begin{array}{c}\text { Branch } \\
\text { number }\end{array}$ & $\begin{array}{c}\text { Flow rate in } \\
\text { design } \\
Q_{s j}(\mathrm{l} / \mathrm{h})\end{array}$ & $\begin{array}{c}\text { Opening value in } \\
\text { design } \\
x\end{array}$ & $\begin{array}{c}\text { Flow } \\
\text { coefficient } \\
K\end{array}$ & $\begin{array}{c}\text { Differential } \\
\text { pressure } \\
\Delta P(\mathrm{kPa})\end{array}$ & $\begin{array}{c}\text { Actual flow } \\
Q(1 / \mathrm{h})\end{array}$ & $\begin{array}{c}\text { Relative } \\
\text { error }\end{array}$ \\
\hline 1 & 400 & 2.30 & 109.743 & 15.1 & 426.45 & $6.6 \%$ \\
2 & 400 & 2.26 & 106.802 & 15.4 & 419.12 & $4.8 \%$ \\
3 & 400 & 2.22 & 103.890 & 16.8 & 425.82 & $6.5 \%$ \\
4 & 900 & 2.65 & 301.835 & 8.6 & 885.15 & $-1.6 \%$ \\
5 & 900 & 2.30 & 241.298 & 15.5 & 949.99 & $5.6 \%$ \\
6 & 900 & 2.24 & 231.332 & 16.3 & 933.96 & $3.8 \%$ \\
\hline
\end{tabular}

\subsection{Analysis of results}

It can be seen from the data in table 2 that after the balance valve of each branch is adjusted to the calculated value obtained by the calculation program, the flow of each branch is basically consistent with the flow under the design condition, and the relative error is less than $10 \%$. Therefore, it can be seen that this hydraulic balance debugging method is effective.

\section{Conclusion}

1) Based on the theory of fluid mechanics and fluid network analysis, this paper proposed a simple and feasible hydraulic balance debugging method for air conditioning water system, and verified the feasibility of this method through experiments. The calculation program is written by MATLAB software to make the complicated calculation steps simple. You only need to manually record simple data such as nodes, branch numbers, differential pressure, and opening value of balance valve, and then enter them into the specified table. The calculation program passes the reading of the table data can automatically complete all calculation steps and directly obtain the opening value of the balance valve of each user branch under the design conditions. The whole debugging process is simple and fast.

2) In the past, various hydraulic balance debugging methods, such as the proportional method and the compensation method, required multiple groups of testers to repeatedly debug the balancing valve of each branch, which consumed a large of time and efforts. The method proposed in this paper has low technical requirements for operators and simple operation. Each branch balancing valve only needs to be adjusted once, which can save a lot of time and efforts.

\section{Acknowledgments}

This work was financially supported by the National Key R\&D Program of China (No. 2018YFC0705005). 


\section{References}

1. Duan qingjin. Discussion on hydraulic balance debugging technology of hvac [J]. Enterprise guide,2016(13):96.

2. Duan qingjin. Discussion on hydraulic balance debugging technology of hvac [J]. Enterprise guide,2016(13):96.

3. Wang yun. Experimental study on the application of balance valve in heating and air conditioning system [D]. Hebei university of engineering, 2016.

4. Hegberg M C. Control Valve Selection for Hydronic Systems[J]. Ashrae Journal, 2000, 42(11):pages. 33-40.

5. G. Henze, C. Felsmann, G. Knabe, Evaluation of optimal control for active and passive building thermal storage, International Journal of Thermal Sciences 43 (2014) 173-183.

6. M.J. McCabe, N. Wang, Commercial Building Energy Asset Rating Program- Market Research, PNNL-21310, Pacific Northwest National Laboratory, Richland, WA, 2012. 